\title{
Kontinuierliche Thoraxkompression bringt keinen Vorteil
}

Fragestellung: Unterscheidet sich bei außerhalb des Krankenhauses durchgeführter kardiopulmonaler Reanimation der Outcome von Patienten, die mit durchgehenden Thoraxkompressionen und überlappender Überdruckbeatmung behandelt wurden, von dem der Patienten, die das bewährte, diskontinuierliche Konzept aus Zyklen von 30 Kompressionen und zwei Ventilationen erhielten?

Hintergrund: Während der kardiopulmonalen Reanimation führt jede Unterbrechung der Thoraxkompressionen zu einer Reduktion des kardialen und zerebralen Blutflusses und somit möglicherweise zu einem schlechteren Outcome. In der vorliegenden Studie wurde mittels kontinuierlicher Thoraxkompressionen die kompressionsfreie Zeit während der Reanimation reduziert und der Einfluss auf den Outcome im Vergleich zum bisherigen Konzept untersucht.

Nichol G, Leroux B, Wang H et al; for the ROC Investigators. Trial of Continuous or Interrupted Chest Compressions during CPR. N Engl J Med 2015; 373: $2203-14$
Patienten und Methodik: In dieser Cluster-randomisierten Studie im Crossover-Design erhielten Patienten mit nicht traumatisch-bedingtem Herzstillstand während der kardiopulmonalen Reanimation entweder kontinuierliche Thoraxkompressionen mit asynchron paralleler Beatmung (Interventionsgruppe) oder 30 Kompressionen im Wechsel mit zwei Ventilationen (Kontrollgruppe). Primärer Endpunkt war das Überleben bei Krankenhausentlassung. Sekundärer Endpunkt war das Erreichen eines günstigen funktionellen Outcome bei Entlassung (modified Rankin Scale 0 -3).

Ergebnisse: Insgesamt wurden 23.711 Patienten randomisiert. Patienten aus der Kontrollgruppe erreichten nach extern begonnener Reanimation signifikant öfter das weiterbehandelnde Krankenhaus (Intervention: 3.108/12.653 (24,6\%) gegenüber Kontrolle: $2.860 / 11.058$ (25,9\%); $\mathrm{p}=0,03)$. Die Überlebensrate nach Entlassung war vergleichbar (Intervention: 9,0\%, Kontrolle: 9,7\%; $=0,07$ ). Ein günstiger funktioneller Outcome bei Entlassung wurde bei $7 \%$ der Patienten der Interventions- und 7,7 \% der Kontrollgruppe erreicht $(p=0,09)$. Das krankenhausfreie Überleben war in der Kontrollgruppe signifikant länger (Intervention: 1,3 $[ \pm 5,0]$ Tage, Kontrolle: $1,5[ \pm 5,3]$ Tage; $\mathrm{p}=0,004$ ).

Schlussfolgerungen: Bei Patienten mit Reanimation außerhalb des Krankenhauses wies die kontinuierliche Thoraxkompression im Vergleich zu dem aktuellen Konzept keine Vorteile auf.

\section{- Kommentar von Stefan T. Gerner und Hagen B. Huttner, Erlangen \\ Aktuell kann dieses Konzept nicht empfohlen werden}

Die Studie konnte trotz hoher Fallzahl keine Vorteile einer kontinuierlichen Thoraxkompression während der kardiopulmonalen Reanimation nachweisen. Das ist interessant, da kleinere Observationsstudien einen Vorteil vermuten ließen. Aus pathophysiologischer Sicht ist der initiale Erhalt der kardialen und zerebralen Perfusion essenziell für den Reanimationserfolg. Insbesondere der koronare Perfusionsdruck nimmt bei Unterbrechung der Thoraxkompressionen rapide ab. Daher erschien es sinnvoll, die kompressionsfreien Intervalle, die bereits durch Rhythmuskontrollen und gegebenenfalls Defibrillationen nötig sind, nicht durch eine Unterbrechung für Ventilationen weiter zu verlängern. Dennoch waren die Mortalität und der funktionelle Outcome in beiden Gruppen vergleichbar. Einzelne Parameter wie das Erreichen des Krankenhauses und das krankenhausfreie Überleben danach lassen sogar einen Benefit des bewährten Konzepts 30:2 vermuten. Neben methodischen Problemen der Studie, wie die Vernachlässigung der klinischen Behandlung nach der Reanimation sowie designbedingte Unterschiede der untersuchten Kohorten, könnte auch das Beatmungskonzept in der Interventionsgruppe relevant gewesen sein; die kontinuierliche Überdruckventilation mit einer Frequenz von 10/min könnte eine verstärkte intrathorakale Druck- erhöhung und somit eine Abnahme der Vorlast bedingt haben. Ferner hatten bisherige Studien postuliert, dass sich eine übermäßige Sauerstoffversorgung in der frühen Reanimationsphase über verstärkte Radikalbildung negativ auswirken und eine passive Sauerstoffversorgung insbesondere in den ersten $\mathrm{Mi}$ nuten einer Reanimation ausreichend sein könnte.

Aktuell kann das untersuchte Konzept von kontinuierlichen Thoraxkompressionen mit Druckbeatmung nicht empfohlen werden. Gegebenenfalls kann eine erneute Studie mit kontinuierlichen Kompressionen - überlappend mit passiver Sauerstoffinsufflation - erwogen werden.

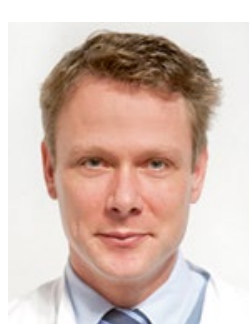

Prof. Dr. med. Hagen B. Huttner, Erlangen

Universitätsklinik für Neurologie, Erlangen E-Mail: hagen.huttner@uk-erlangen.de 